\title{
A SMART Brain Controlled WHEELCHAIR BASED MICROCONTROLLER SYSTEM
}

\author{
Sherif Kamel Hussein Hassan Ratib \\ Associate Professor- Department of Communications and Computer Engineering, \\ October University for Modern Sciences and Arts, Giza, Egypt \\ Head of Computer Science Department - Arab East Colleges - Riyadh- KSA
}

\begin{abstract}
The main objective of this paper is to build Smart Brain Controlled wheelchair (SBCW) intended for patient of Amyotrophic Lateral Sclerosis (ALS). Brain control interface (BCI) gave solutions for a patients having a low rate of data exchange, alsoby using the BCIthe user should have the ability to meditate and tension to let the signal get received. Using the BCI continuously is very much exhausted for the patients, Theproposed system is trying to give all handicapped people and ALS patients the simplest way to let them have a life at least near to the normal life. The system will mainly depend on the Electroencephalogram (EEG) signalsand also on the Electromyography (EMG) signals to put the system in command and out of command. The system will interface with user through a tablet and it will be secured by sensors and tracking system to avoid any obstacle. The proposed system is safe and easily built with lower cost compared with other similar systems.
\end{abstract}

\section{KEYWORDS:}

Smart Brain Controlled wheelchair (SBCW), Amyotrophic Lateral Sclerosis (ALS), Brain control interface, Brain Computer Interface (BCI), Electroencephalogram EEG, Electromyography EMG

\section{INTRODUCTION}

A person with physical disabilities can move from one place to another independently with the help of wheelchair having joystick, touchpad, keyboard etc. But people who lose their muscles control are unable to operate the wheelchairs. Brain computer interface (BCI) is a latest method of communication between computers and human being. It uses a direct way of access to the intentions of a person. The communication towards computer and the will of the person which is fed into the machine gets collected at its source, the brain[1].

Brain controlled wheelchair is a wheelchair serving full paralyzed patient. Mainly it serves amyotrophic lateral sclerosis patients. The brain controlled wheelchair will be consisted of sensors that receive certain signals from the brain or from the voluntary actions done by the patient. The control unit will read those signals and transmit them to the motor that makes the wheelchair move in certain directions according to signals received [2-5].

The use of human brain signals to control devices and software in order to interact with the world is an important problem in bioengineering. The solution to this problem includes two stages. The first stage is the development of the interface between the brain and computer. The second stage is the design of brain based control of devices. The basic aim of the BCI is related to the design of communication channel for disabled people. A BCI system provides communication between computer and mind of disabled people. This communication can be based on muscular movements during brain activity or the changes of the rhythms of brain 
signals. These brain activities can be estimated with electroencephalographic (EEG) signals. Since the brain signals are very weak, it is requested to apply some spatial and spectral filters and amplifiers to the EEG to extract characteristic features of signals. Several EEG signals can be detected, resulting in different types of BCI. These signals are based on change of frequencies, change of amplitudes. For example during voluntary thoughts the frequencies of signals are modified, during movement a synchronization / desynchronization of brain activity which involves rhythm amplitude change. This relevant characteristic makes rhythm based BCI suitable to be used [6].

The main idea of designing a Brain Controlled Wheelchair (BCW) as shown in Fig.1 is have a signal from the brain and to transfer it to a motion using the Brain Computer Interface (BCI). The (BCI) is the electrical action of the cerebrum that can be observed by utilizing a variety of electrodes to be set on the patient scalp to get the EEG signals.

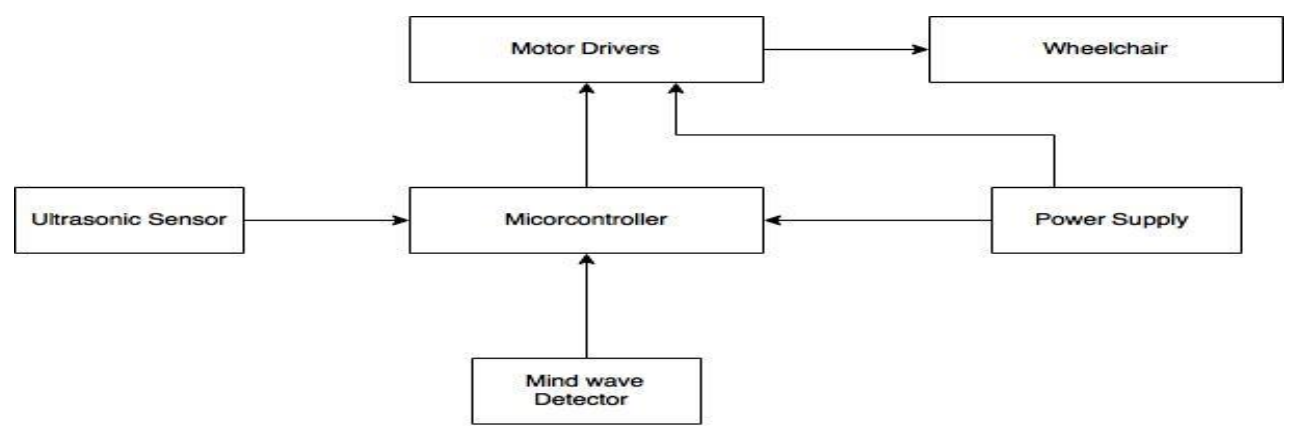

Figure 1. Conceptual Diagram of System

\section{LITERATURE SURVEY}

The BCI is framework that gains and analyzes EEG signals with the objective of making an immediate high-frequency connection between the mind and the controller. BCI is such a noninvasive technique after being developed from transplanting parts in heads of humans or animals. Instead of transplanting, nowadays, an array of electrodes is set on head of the patient to monitor the electricals activity of the brain. This process is known as electroencephalography (EEG) that is mainly used in the most common systems in addition some systems also use the electrooculogram (EOG) and the electromyogram (EMG) as assistive systems.

\subsection{First Brain Controlled Wheelchair Using Only BCI [7]}

Kazuo Tanaka was the first one who used BCI in controlling a wheelchair in 2005. The aim is to control the movement and directions of an electric wheelchair using only EEG signals. The experiment system comprises of an electro cap (cathodes), EEG (amplifier), an electrodes box, a simple to-computerized analogue to digital converter, and a PC. This system was depending on continuous commands given by the user (not paralyzed person.

\subsection{Hand-Free Wheelchair Control with EMG and EOG [8]}

In 2007 Chun.S.L. and his team developed a wheelchair without hands control system by utilizing EMG signals to detect the required direction and EOG signals to control the speed of a powered wheelchair. EMG and EOG signals are gained by utilizing a Cyber link, and are utilized to create controls orders. They are additionally used to convert the framework between (in control) and (not in control ) states. At the point when (not in control) express, the patient could do his/her required subject or other action with no stressing over movement to activate the 
control commands. The framework is of minimal effort, simple to setup, and simple to utilize. User may require about thirty minutes of training with a test system to control the framework voluntarily before utilizing the genuine wheelchair.

\subsection{BCW Design Based P300 and Path Guidance [9]}

The wheelchair that is based on P 300 interface technique is a slow but safe. The experiments applied on that system proved that the wheelchair could be safely controlled in indoor environment. An EEG BCI is used to receive signals from the brain by putting electrodes to the user's head. P300 design BCI is utilized where described by error rates of $3 \%$ by reaction time up to seconds. So as to simplify the movement controlling, the wheelchair is compelled to move through ways that is predefined in programming enlisted areas. The controlling ways can be gone into the framework utilizing a basic and proficient way editorial manager, which likewise empower assistants to adjust the BCW changing natural conditions. The user assignment then just comprises of choosing the goal and managing surprising circumstances through a discourse plot. Along these lines the framework requires at least information and focus from the user.

P300 design is a very much examined and stable contrast at the focal locales of the EEG estimations relating to the uncommon or rare occasions. An arrangement of things is shown on a LCD screen and flashed every one after the other for every request, with just a single of them the objective being applicable for the user. The patient is told to concentrate his/her consideration on the required objective. P300 is the measuring of astonishment, and not visual flag. A positive potential regularly happens around 300 milli-seconds after an uncommon occasion, from which the objective can be resolved.

The user is not required to get trained in order to be able to use this system and only a couple of minutes are enough for the user to get trained. Moreover, the other techniques are not as simple as this system for users in training. Concluding, the advantage of P 300 guided path is safe for users even though it is slow; but its main problem is that the signal is occupied with noise also the rate of transmitting the information is low. Unfortunately, the user will not be able to use a wheelchair with such a system as it cannot move in a free environment (it only moves within certain area as mentioned before by a guided path). As the system requires an indoor GPS makes this system unsuccessful one due to the high cost which is not preferred

\subsection{Robotic Architecture [10]}

Since patients are physically unfit to control the seat utilizing an ordinary interface or in light of the fact that they are regarded unequipped for securely driving them. So a robotic architecture brain-controlled wheelchair was invented in March 2013. This wheelchair had an additional feature to that P300 one. Since the P300 was very exhausting to the user by performing a lot of mental tasks in order to reach certain destination. Even though the mapped P300 wasn't that much useful to be used outside certain workspace. This robotic architecture wheelchair was designed to move anywhere in all directions till the user orders to stop. Then again, another BCI wheelchair, which is likewise in light of the P300 worldview, does not limit the client to exploring in known pre-mapping situations. Rather, in the following design, the client can choose sub goals, (for example, left or right or mid-ahead, and so on).

\subsection{Serial interface to control a two-class of imagery-motor wheelchair [11]}

A proposition is to control a wheelchair by a Brain-Computer Interface in view of the separation of just two mentally assignments. Wheelchair uprooting is working with discrete developments. The controlled signals utilized are sensor motor rhythms regulated through the 
right-handed engine symbolism undertaking or mentally sit without moving the state. Eccentricity of controlling framework is that the system depends on a sound-related interfacing that gives the client all the four route summons. The utilization of the two mental assignments to choose orders maybe encourage controlling and diminish mistake rate contrasted with different control frameworks for wheelchairs.

Mainly the BCW has only two modes NC (not generating any command) and the IC (intentional control) and four commands (forward, backward, left and right), to change the mode from NC to IC the user should declare in his visual glass he/she should move a laser bar to reach above $70 \%$ in the direction of the intentioned mode, but if the user couldn't reach the required mode so the BCW will keep on the last mode. The two tasks needed from the user mentally are to just declare if he/she wants to move forward or backward and the direction could be chosen by only giving the laser bar the direction with his/her eyes depending on the special navigation system programed especially for this $\mathrm{BCW}$.

The main advantage of this system is the custom made control unit. Also the navigation system which will help the user to avoid obstacles virtually. Also the NC mode that will help the user to do his own mind work without worry about the motion of the wheelchair

The disadvantage of the system is that the system has an old control system depends on the joystick and also the user should declare either to work with the BCI system or the normal system, the system is not ready to be used by any kind of patient with a SMR error less than $70 \%$. The destruction done by the glasses because of the laser bars appear in front of the user may prevent the patient from reaching the aimed goal. The cost of such system will be very high so that it will never be available for mass production.

\subsection{Brain-Controlled Electric Wheelchairs [12]}

The system depends on using the Neuro Sky that record brain signals by biosensors. The signals are EEG from the brain activity and EMG from the facial activity. It captures the brain signals and send them to the android application to control the movement and the directions of the BCW. Android application get the EEG and EMG using the NeuroSky by Bluetooth. The signals are proceeded in the android application through algorithms and then the signals will be sentto control the $\mathrm{BCW}$ through the Bluetooth module that attenuate the signal .Control unit consist of Arduino microcontroller to control the motions and the directions of the BCW as shown in figure 2 .

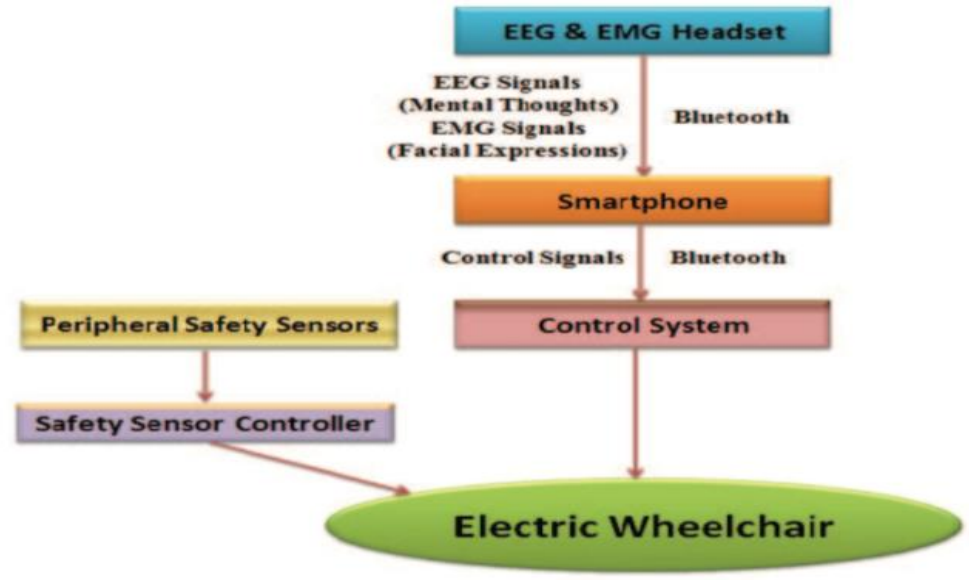

Figure 2. System block diagram 
Mainly the advantage of the system is using a simple component to reach an amazing result as mainly it is the least cost in all the systems with the highest results. Using the Arduino and the ultrasonic sensors reduce the cost of the $\mathrm{BCW}$ more than the half. Also the efficiency of the Arduino is much better than any other controller because it works in series to send and receive the signals which make it work in high processing.

The system considered as one of the most successful systems compared with all other pervious systems because the percentage of the success the system reach, but the system have two main disadvantage; the first one is that the sensors are connected on a separate controller which is not necessary as the Arduino can do both functions, checking the signals received from the Bluetooth module and also the sensor signals. Secondly having a stop command between every and each command make the user get tired because the time taken to reach the aim is too long.

\section{The NeWly Proposed System}

\subsection{System Block Diagram}

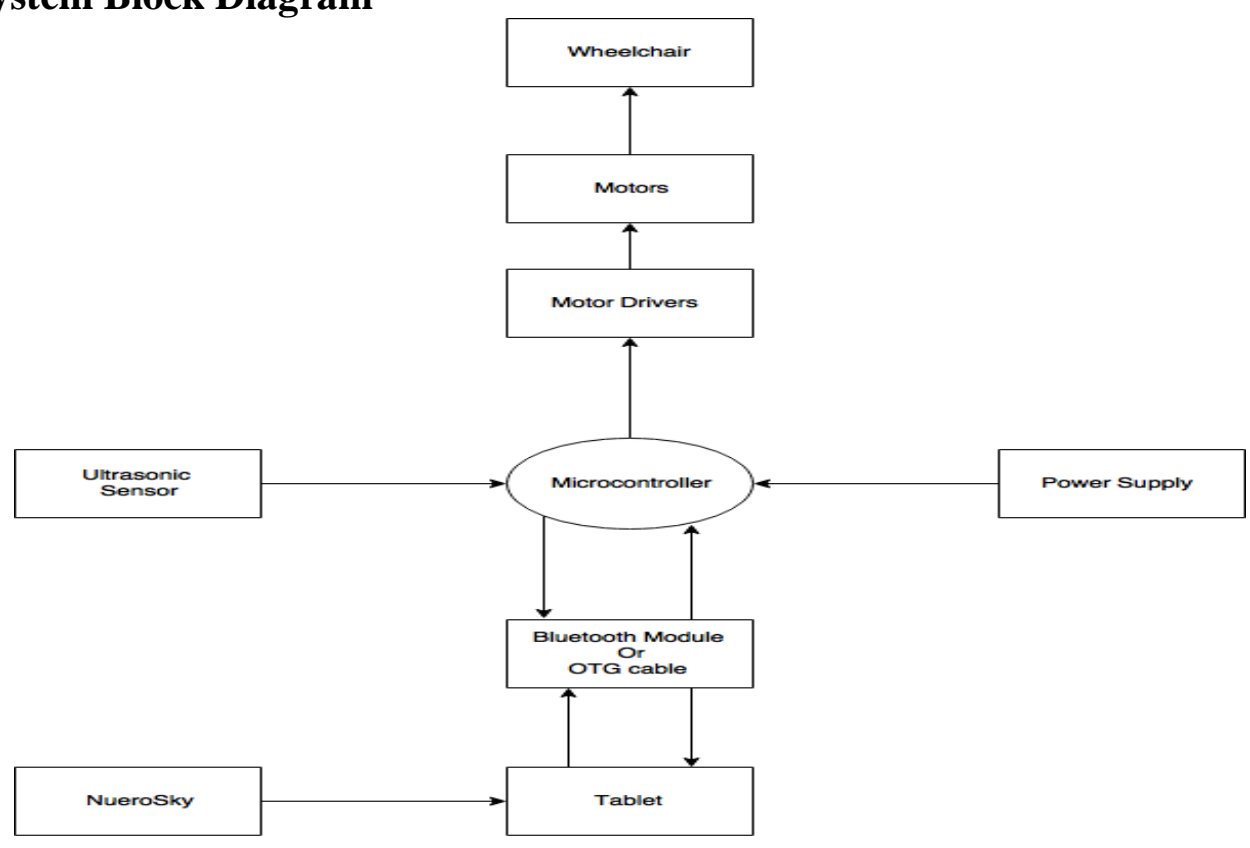

Figure 3. Block diagram description

\subsection{System Description}

\subsubsection{Mind Wave}

Reading your mind sounds very freaky. A few cerebrum checking systems like fMRI, PET, or EEG are fit to break down what you are considering, imagining, or seeing. fMRI and PET are not common used because they depend on physical change to read the mind waves as blood pressure. EEG is regularly noninvasive, with the anodes put along the scalp, albeit other cathodes are here and there utilized as a part of particular applications. EEG measures the voltage changes coming because of the ionic current inside the cells of the cerebrum. EEG convert the brain signals to electrical signals, as it is recorded from numerous terminals mounted on the head as shown in Figure 4. The equation used to transform the raw values of EEG to voltage is

$$
\frac{\text { rawvalue } \times \frac{1.8}{4096}}{2000}
$$


International Journal of Artificial Intelligence \& Applications (IJAIA) Vol.10, No.5, September 2019

- Delta

(1-3Hz): sleep

- Theta

(4-7Hz): relaxed, meditative

- Low Alpha (8-9Hz): eyes closed, relaxed

- High Alpha(10-12Hz)

- Low Beta (13-17Hz): alert, focused

- High Beta $(18-30 \mathrm{~Hz})$

- Low Gamma (31-40Hz): multi-sensory processing

- High Gamma (41-50Hz)

Figure 4. Types of mind wave

NeuroSky Sense meters comprises of a headset, ear-cut, and a sensor arm. The headsets consist of reference and ground cathodes set to the ear cut and the EEG anode is put on the sensor arm, which lay on the temple on the eye as appeared in figure 5, NeuroSky headset is a Bluetooth device empowered headset with a solitary, biosensor where distinguishes black out (EEG and EMG) passive.

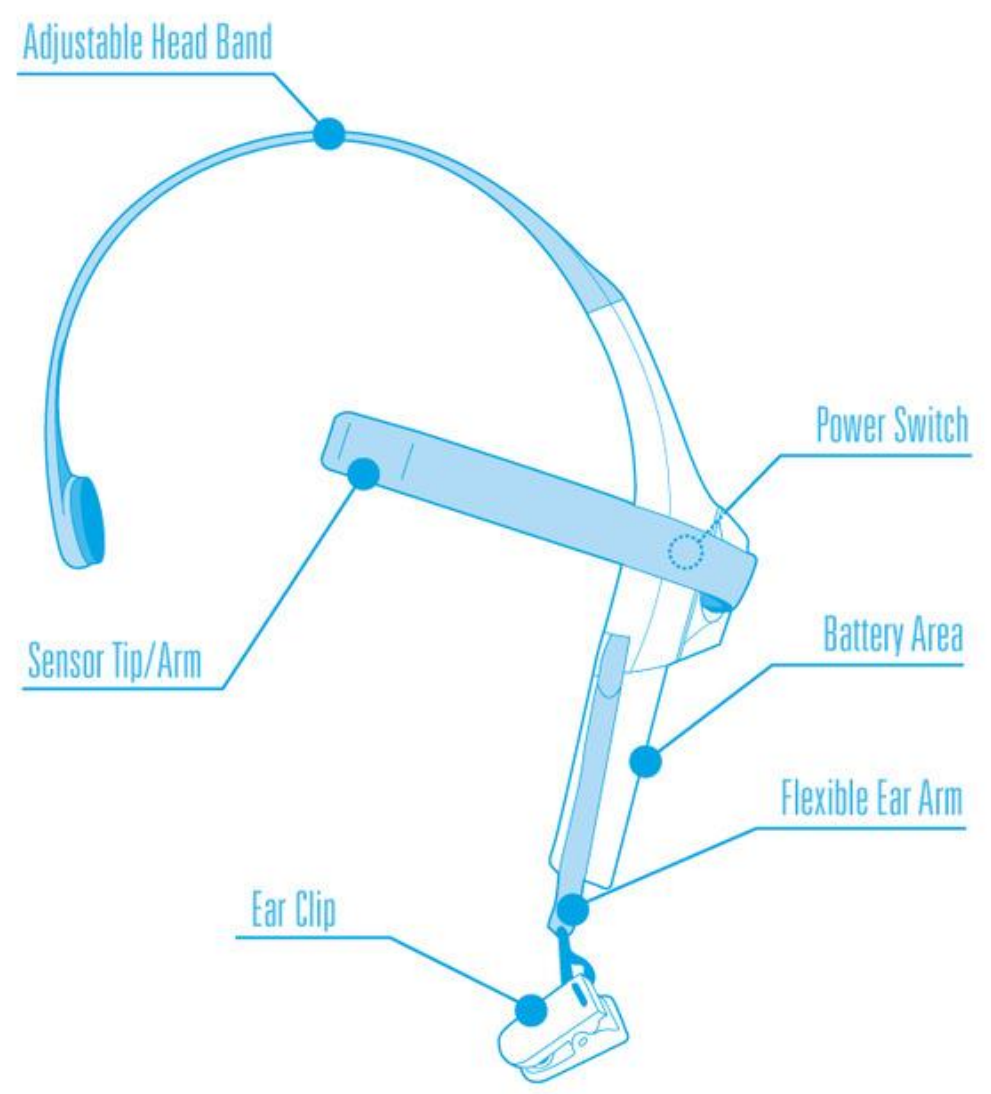

Figure 5. NeuroSky components

\subsubsection{Mobile Devices}

The mobile device is mainly used to receive the signal from the EEG and send the decision of the patient to the microcontroller through the Bluetooth module. The most common used mobile devices worldwide are the android systems mobile that is because the android system is an open 
source system where every company and person can update it to use the software in the way they need it, although it is easy to get the approval to launch an application for the android system comparing by any other system.

\subsubsection{Bluetooth Module}

It consists of four ports, Vcc, GND, Tx and Rx, The Vcc is the power supply taken from the controller equal to $3.3 \mathrm{v}$ while GND is the ground with zero volt, the Tx is responsible for transmitting data while the Rx is to receive data, The Tx should be connected to the Rx in the Arduino while the $\mathrm{Rx}$ should be connected to the Tx in the Arduino. The main task of the Bluetooth module is to work in serial communication between the Arduino and the Application on the mobile device. The application is mainly designed for sending and receiving orders to the Bluetooth module.

\subsubsection{USB On-The-Go (OTG)}

USB On-The-Go (OTG) is an institutionalized detail for USB associations that enables a gadget to peruse information from a USB connection without requiring a PC. The gadget is essentially turning into a "USB Host", which isn't a capacity each gadget has. It adds an additional stick to the miniaturized scale USB attachment. In the event of plugging an ordinary A-to-B USB link, the gadget demonstrations in fringe mode.

\subsubsection{Power Supply}

There are different batteries could be used in the electrical wheelchair. Wet Battery is the chosen one to be used with the proposed system.

\subsubsection{Wet Battery}

Mainly it depends on the chemical reactions with the sulphuric acid and it leadsto obtain an electrical energy .The batteries should be filled with distilled water.

Positivity:

- Cheaper than other types

- Not very able to get overcharging

- Long work time with low maintenance

Negative Aspects:

- maintenance is required

- leakage of battery acid

- high rate of discharging

- cannot travel in airplanes

\subsubsection{Sensors:}

The patients that need such proposed systems don't always aware by every obstacle they face so they need the support to recognize every single step and assure it is safe to be done.The sensor is mainly used to stop the wheelchair or change its direction if the chair is going to hit something before the patient give a command to avoid it. There are two common used sensors to do such task; Laser Mapping Sensor and Ultrasonic Sensor. 


\subsubsection{Laser Mapping Sensor (LIDAR)}

The idea of the LIDAR working is very simple just emit light and measure the time it takes to reach back the source of emission. The equation of the distance is:

$$
\text { Distance }=(\text { Speed of Light } * \text { Time of Flight }) / 2
$$

The main advantage of (LIDAR) is that it can cover over than 150,000 points in the same altitude so it is very difficult to fail in recognizing any obstacle. Also the (LIDAR) is too much expensive, it may cost $150 \$$ per only one LIDAR.

\subsubsection{Ultrasonic Sensor}

The ultrasonic sensor sends a high ultrasonic wave and get it back to calculate the distance between the object and the obstacle by using the same equation as the LIDAR. The sensor has double openings ,one to send the ultrasonic wave and other one to get them.

The advantage of using the ultrasonic sensor is mainly about the price where the sensor costs about $8 \$$ and it covers nearly about six meters. The disadvantage of the ultrasonic sensors is the durability where the life time of the sensor is from 1 year to 2 years depending on the using process.

In the proposed system the Ultrasonic sensor will be used because of the reliable price where it is of very low cost compared with others and also it gives the same result and covers the area required.

\subsubsection{Controller}

Usually the used controller in such a systems are either the Programmable Logic Controllers (PLCs) or the Microcontrollers. The PLC is very difficult to be used although it gives a high productivity but also the Wight and the size of PLC is very much bigger than the microcontroller. Table 1 shows the main advantage of the microcontroller compared with the PLC.

Table 1. Comparison between Microcontroller and PLC

\begin{tabular}{|c|c|c|}
\hline & PLC & Microcontroller \\
\hline Cost & High Cost & Reasonable \\
\hline USB Attach & Not available & Available \\
\hline Stability & More Stable & Less Stable \\
\hline Open Source Software & Not an Open Source & Open Source \\
\hline Output Pins & Not Enough & Enough \\
\hline
\end{tabular}

\subsubsection{Microcontroller (Arduino)}

There are a lot of microcontrollers that can be used in such proposed systems, but the one that can deal with a lot of sensors and tasks at the same time will be the Arduino.

The Arduino's energy supply prerequisites are extremely basic; it could be connected to PC or a battery pack, and it will begin running code quickly. In the event that the power is separated, it will stop; there's no compelling reason to run a close down process. To support networks connection an additional Ethernet card should be required. Also for Wi-Fi availability an additional card should be used. The open source Arduino lets the user start coding in an easily way. It could run on different operating systems such as windows, mac ox and Linux. 
International Journal of Artificial Intelligence \& Applications (IJAIA) Vol.10, No.5, September 2019

\subsubsection{Motors}

Motors as used to convert some types of energy to mechanical energy, specifically the electrical motors coverts the electricity to mechanical movements by interacting of the magnetic field with the current carry conductors. The reverse process occurs to gain an electrical energy from mechanical energy by dynamo [13].

\subsubsection{Brushed De Motor}

Figure 6 shows the Components of the Brushed DC Motor used in the proposed system.

Advantages:

- Only two wires control.

- The brushes should extend for a long time.

- Low cost.

- Simple.

Disadvantages:

- Periodic maintenance is required.

- Internal heat formation because of internal router

- Low speed range because of mechanical limits of the brushes.

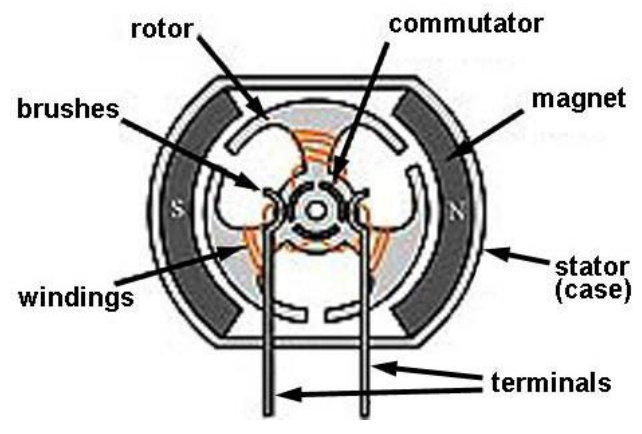

Figure 6. Brushed Motors [13] 
International Journal of Artificial Intelligence \& Applications (IJAIA) Vol.10, No.5, September 2019

\subsection{Flowchart}

Figure 7 shows the flow chart of the proposed system. Depends on the signal received by the Bluetooth module the wheelchair accordingly will start moving forwardly, backwardly, to the right, to the left or stop. The wheelchair will keep moving as long as the distance is greater than or equal $30 \mathrm{~cm}$.

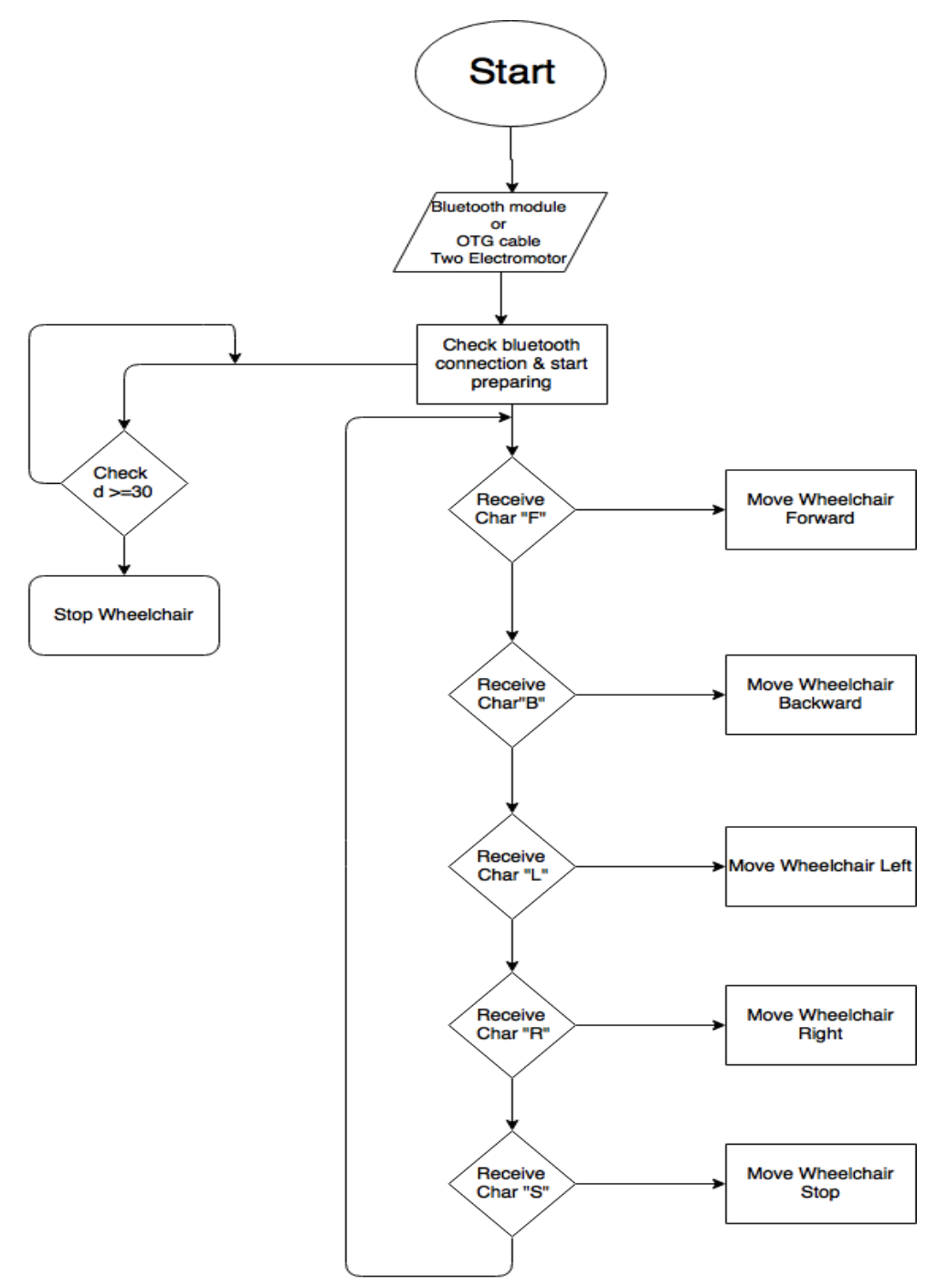

Figure 7. Flowchart

\section{SYSTEM DESIGN AND IMPLEMENTATION}

\subsection{Circuit Diagram}

Figure 8 shows the Arduino and the motor driver .Figure 9 shows the Arduino connected to both motor driver and ultrasonic sensor. 
International Journal of Artificial Intelligence \& Applications (IJAIA) Vol.10, No.5, September 2019
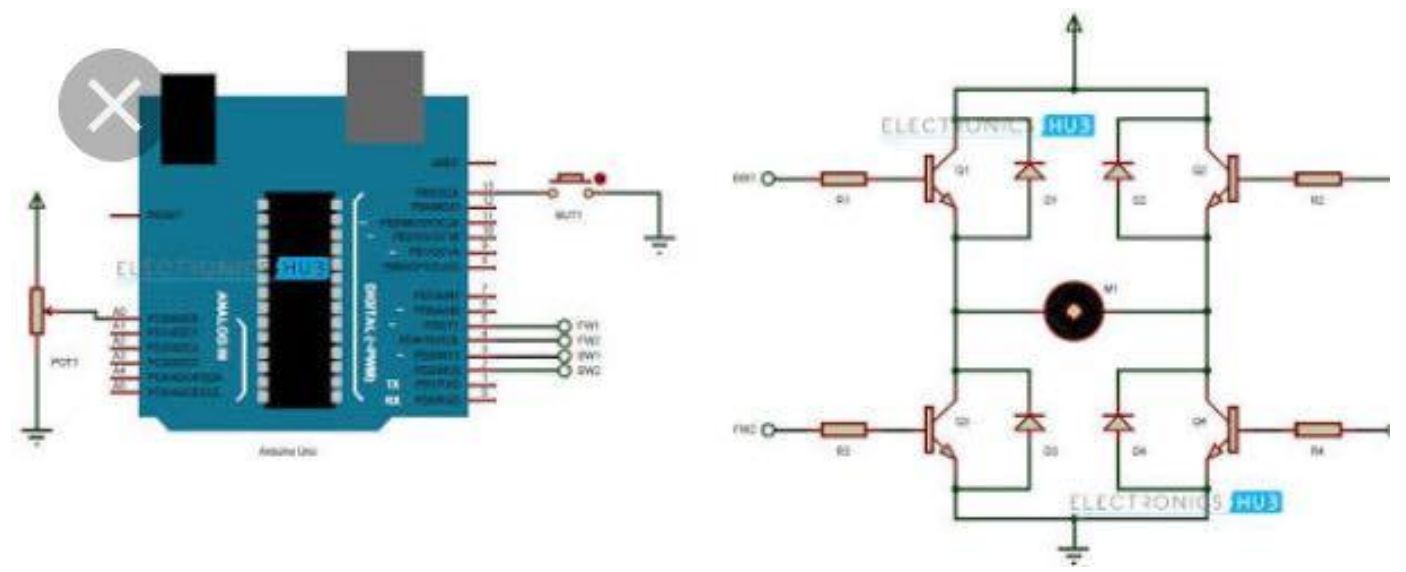

Figure 8. Arduino and motor driver.
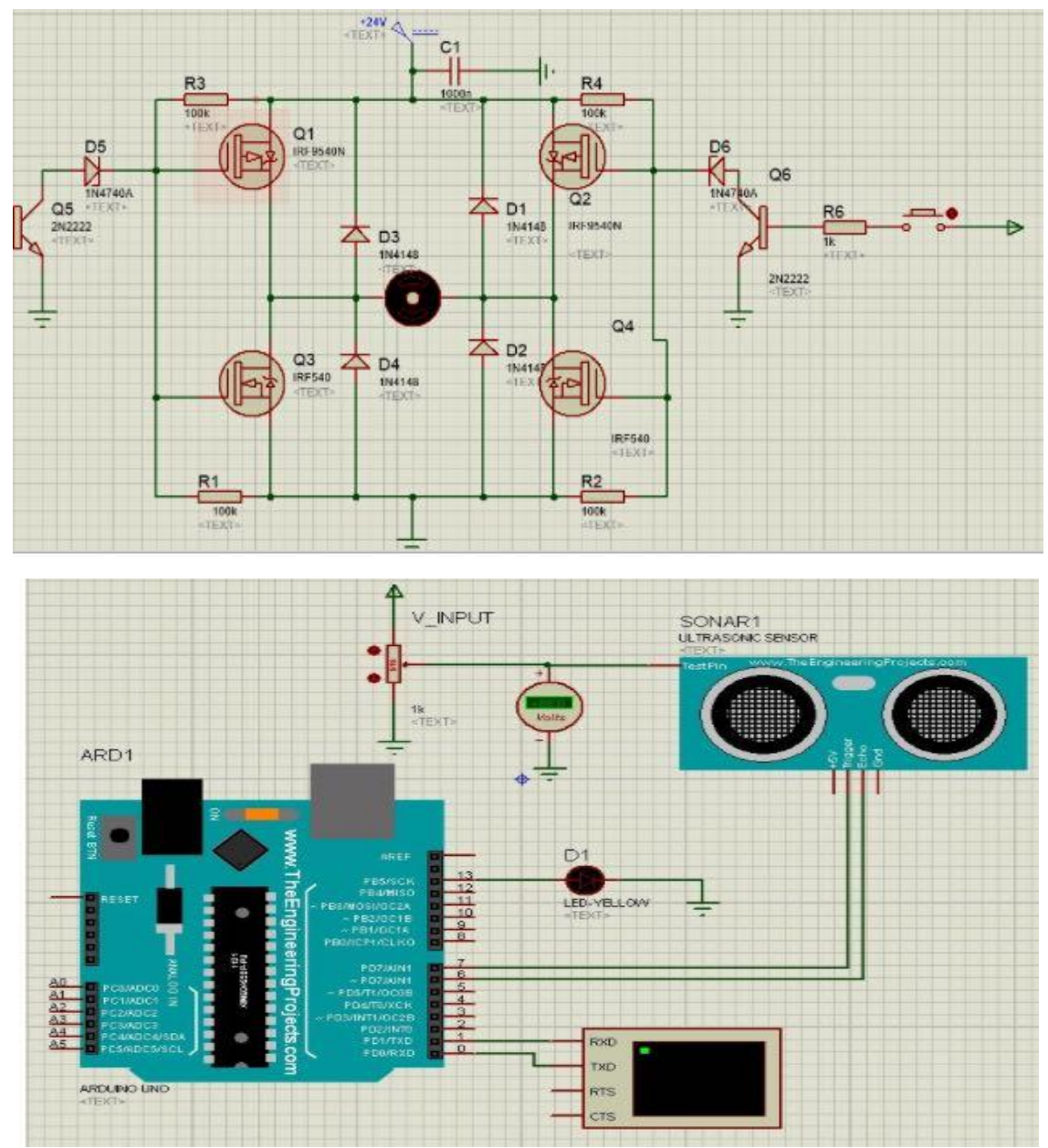

Figure 9. Arduino and motor driver connections.

\subsection{Hardware Implementation.}

At the beginning of the hardware implementation the availability of controlling the two DC motors using H-bridge relays had been tested. Mainly the most important thing was to control the directions of the wheelchair as it is of the main concern. The relays was required to take 
International Journal of Artificial Intelligence \& Applications (IJAIA) Vol.10, No.5, September 2019

command from the Arduino in form of pulse modulation. Also the Arduino is taking the instructions in form of the serial input represented by letters $(\mathrm{F}=$ Forward, $\mathrm{B}=\mathrm{Backward}, \mathrm{R}=$ Right, $\mathrm{L}=\mathrm{Left}, \mathrm{S}=\mathrm{Stop}$ ).Every and each letter is responsible for a move in the direction with the start of the letter.

The Figures $(10-14)$ show the motor movement directions.

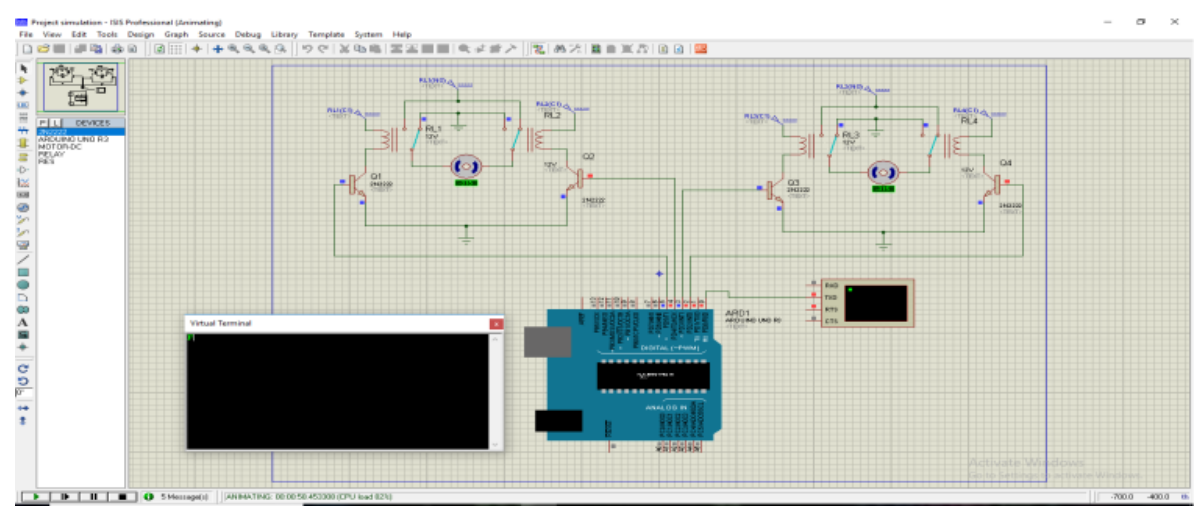

Figure 10. Controlling Motors to move forward

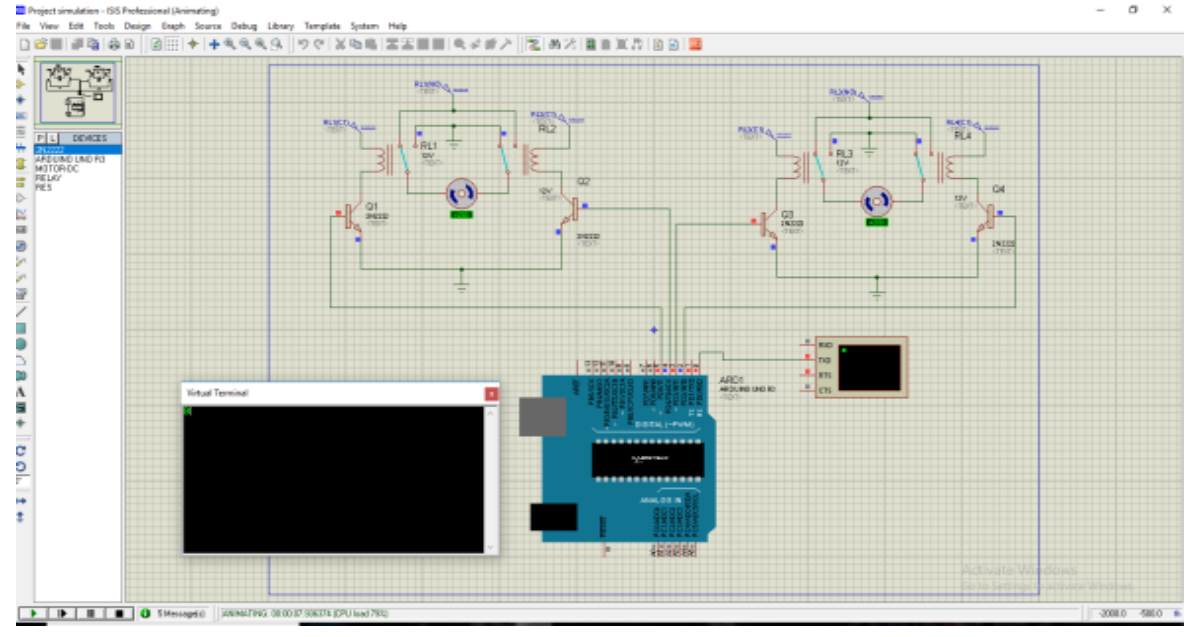

Figure 11. Controlling Motors to move backward

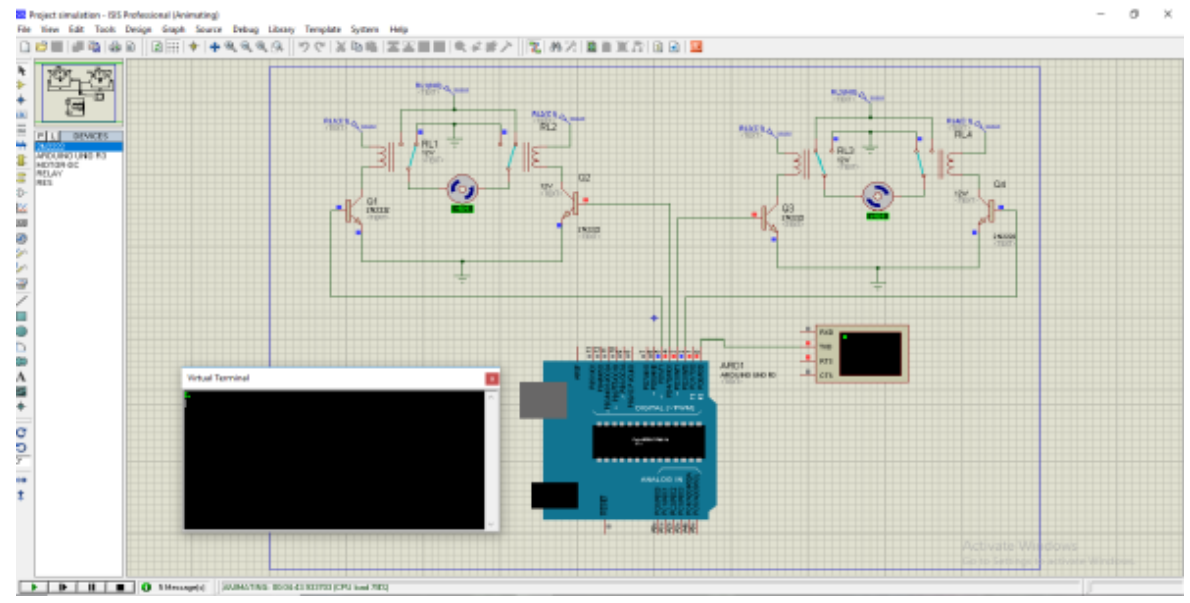

Figure 12. Controlling Motors to move left 


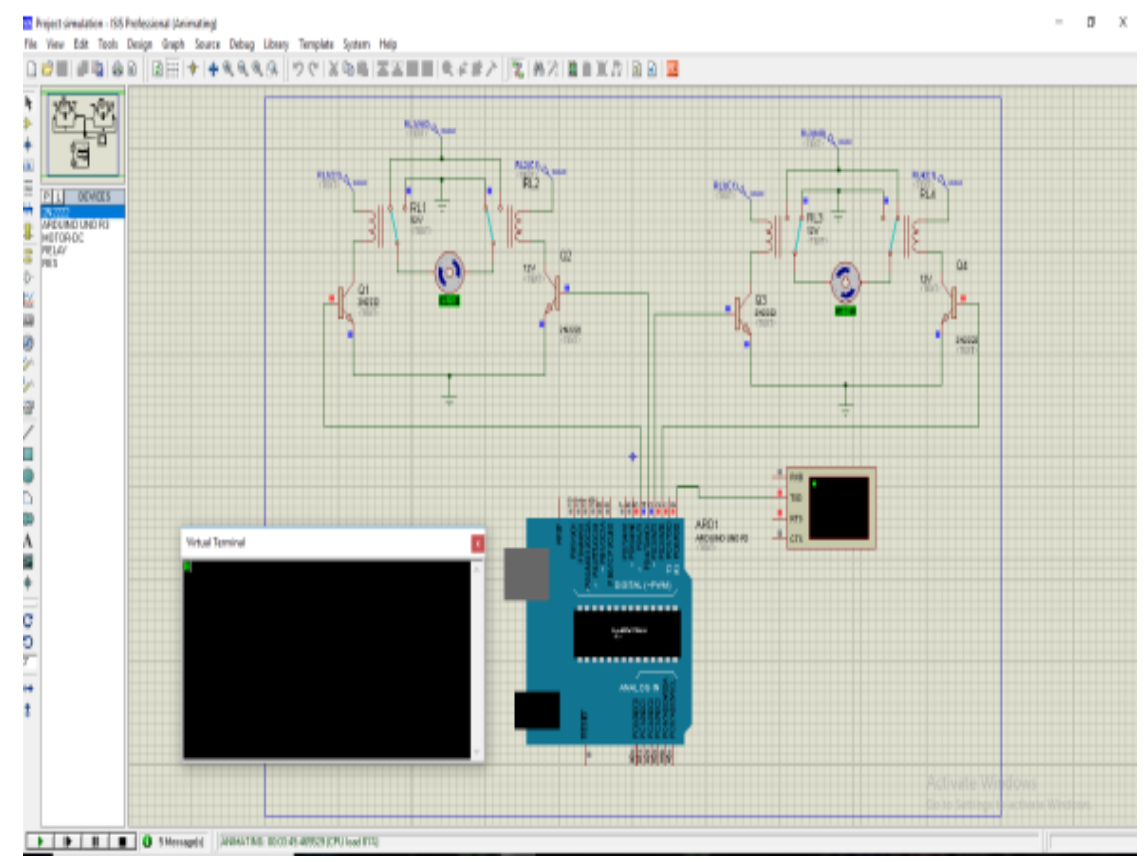

Figure 13. Controlling Motors to move right

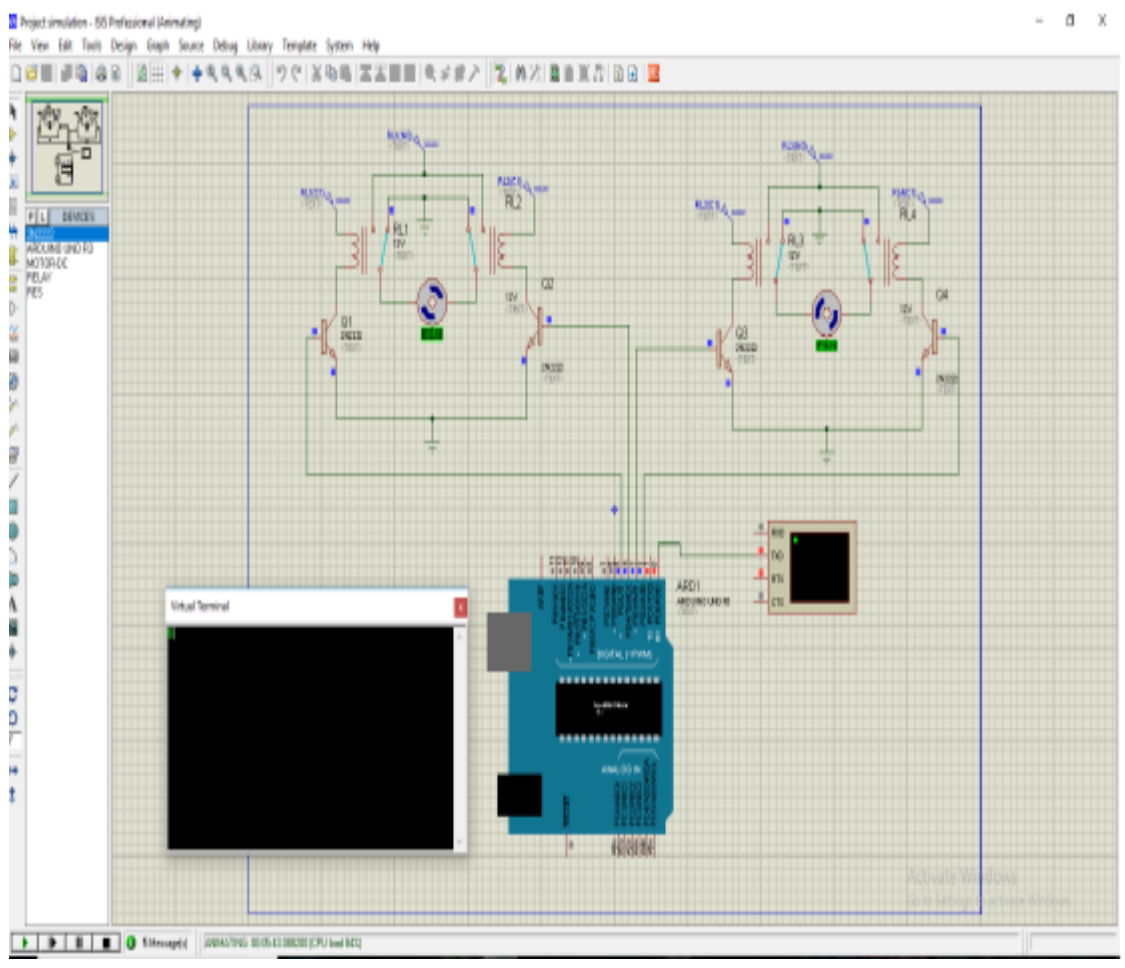

Figure 14. Controlling Motors to stop

DC motor driver (30 amp continuous - 80 amp peak)shown in Fig.15is designed to drive medium to high power brushed DC motor with current capacity up to 80A peak and 30A continuously. Fully NMOS design not only provides faster switching time, but also more efficient and no heat-sink or fan is required. Besides that, the driver also incorporates some user friendly features such as reverse polarity protection and onboard Pulse Width Modulation 
(PWM) generator which allow to operate without a host controller. The motor can simply be controlled with the onboard switches and speed potentiometer. External switches and potentiometer can also be used.

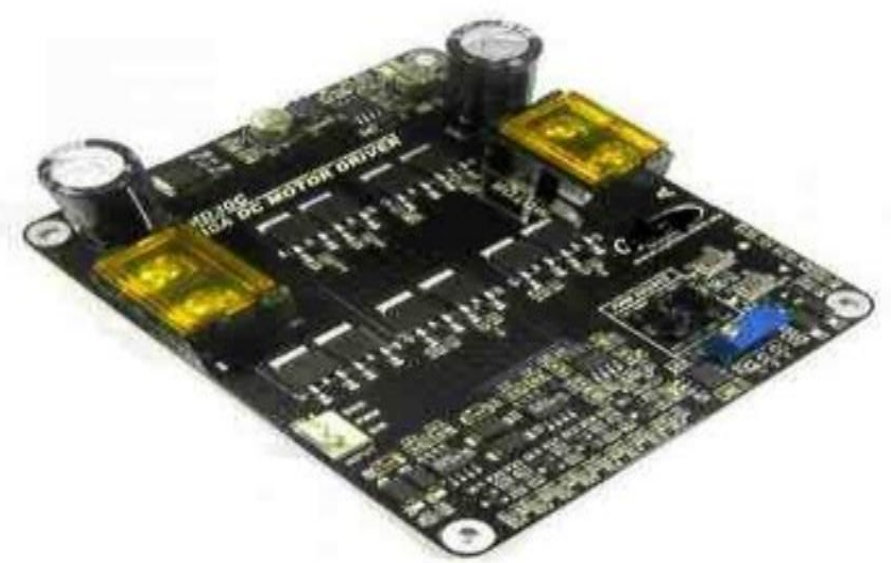

Figure 15. Motor Driver

\subsection{System Software}

Arduino $\mathrm{C}$ code is used for programming the Microcontroller. The interface with the user is not complex. For all cases android studio is suitable for the need to design and operate the application for the tablet. For software, android studio is used for designing the layout of the application used by the user. In the implementation there are five labels. Each label is used for expressing a certain direction, four arrows are there for each direction: one for forward movement, one for backward movement, one for right movements, one for left movements and a stop label for stopping. This application is uploaded to the tablet so as when the tablet receives signals from the NeuroSky mind wave, it gives the users a feedback and a double check for each order. For giving orders concentration is required. the user chooses the lightened label and a blink is for shifting to another label.

\section{SYSTEM RESULTS}

The newly proposed system had been tested by 3 participants with five sessions as shown in Table 2.The results shows the control of the speed and directions of the wheelchair with the chosen components, the wheelchair successfully started the movement from zero speed to the maximum. Also the directions were controlled. The real implementation is shown in Figs.(16$18)$.

Table 2. Results

\begin{tabular}{|c|c|c|c|c|c|c|}
\hline Subject & F & R & B & L & S & Avg. of Success \\
\hline 1 & 8 & 10 & 7 & 9 & 9 & $86 \%$ \\
\hline 2 & 9 & 8 & 10 & 7 & 8 & $84 \%$ \\
\hline 3 & 9 & 10 & 10 & 8 & 9 & $92 \%$ \\
\hline
\end{tabular}


International Journal of Artificial Intelligence \& Applications (IJAIA) Vol.10, No.5, September 2019

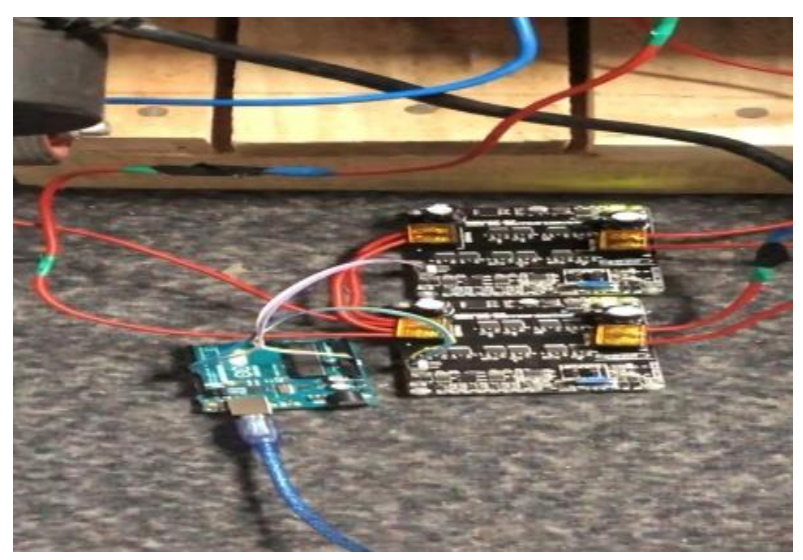

Figure 16. Real implementation photos

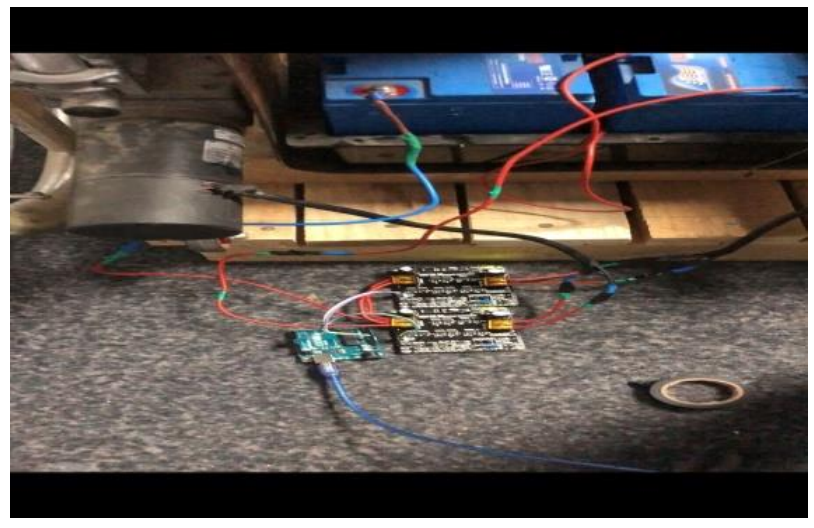

Figure 17. Real implementation photos

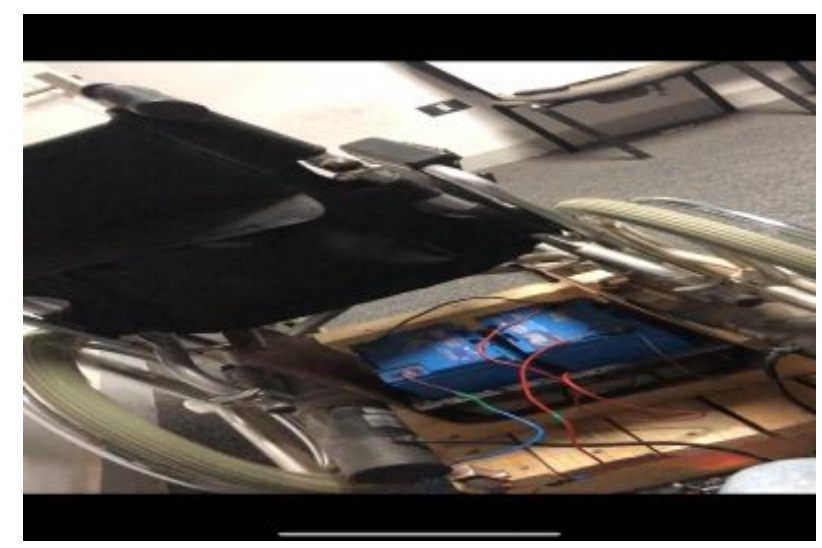

Figure 18. Real implementation photos

\subsection{Design Flow of Brain Controlled Wheelchair}

The workflow gives the step by step process about the brain-controlled wheelchair. Fig. 19 shows the design flow of brain controlled wheelchair. 
International Journal of Artificial Intelligence \& Applications (IJAIA) Vol.10, No.5, September 2019

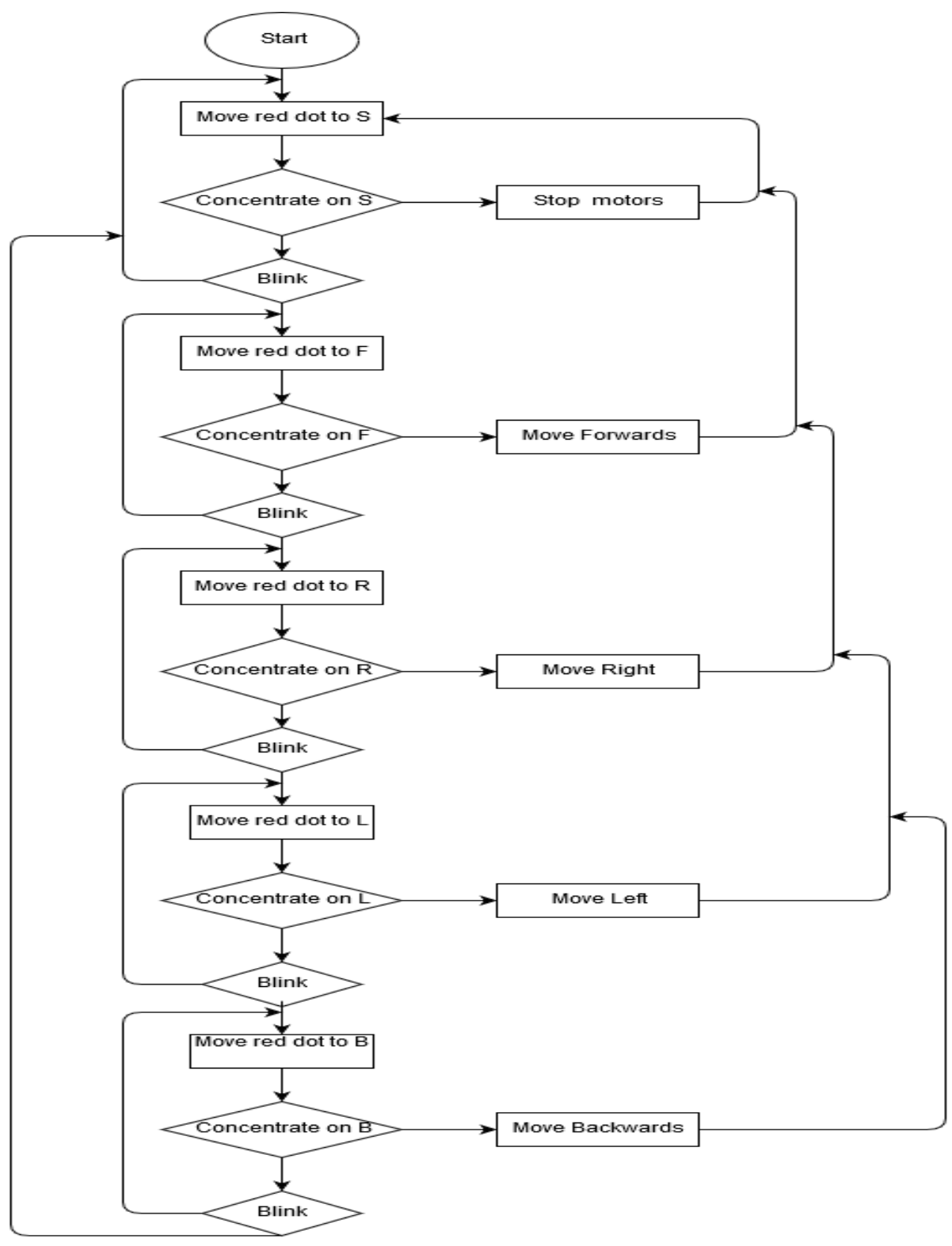

Figure 19. Software flowchart

\subsection{Direction Selection}

Figures (20-24) show different directions layouts

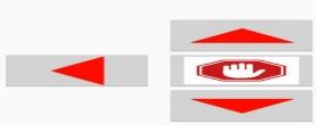

Figure 20. Mobile application layout 
International Journal of Artificial Intelligence \& Applications (IJAIA) Vol.10, No.5, September 2019

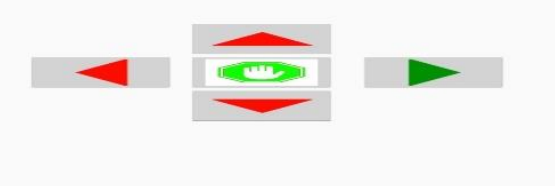

Figure 21. Mobile application layout for right direction

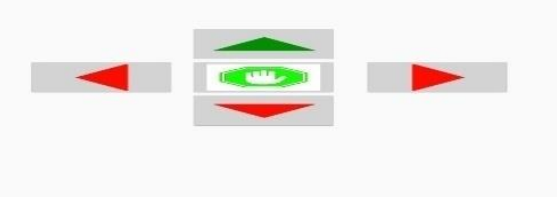

Figure 22. Mobile application layout for forward direction

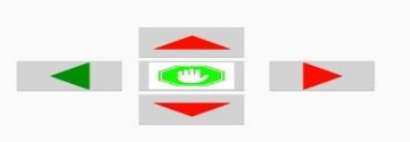

Figure 23. Mobile application layout for left direction

Figure 24. Mobile application layout for backward direction

\section{Cost Analysis}

Table 3 shows the cost per unit and also the Economical Cost for 100 units.

Table 3. Cost analysis

\begin{tabular}{|c|c|c|}
\hline Component & Cost per unit & $\begin{array}{c}\text { Economical cost } \\
\text { for 100 units }\end{array}$ \\
\hline Wheelchair & $145 \$$ & $11,600 \$$ \\
\hline NueroSky & $200 \$$ & $18,000 \$$ \\
\hline Arduino & $30 \$$ & $1000 \$$ \\
\hline OTG-Cable & $5 \$$ & $250 \$$ \\
\hline Ultrasonic Sensor & $12 \$$ & $600 \$$ \\
\hline Motor Driver 30 Amp & $35 \$$ & $3250 \$$ \\
\hline Total & $462 \$$ & $39000 \$$ \\
\hline
\end{tabular}


International Journal of Artificial Intelligence \& Applications (IJAIA) Vol.10, No.5, September 2019

\section{CONCLUSION}

Nowadays a high percent of handicapped may not be able to live their life normally because the disability. It is very difficult for them to move to do their jobs as if their life are postponed. Some also have a diseases as Amyotrophic lateral sclerosis (ALS) that harm all the muscles in the body as the patient cannot move any of his muscles except eye muscles. Many previous brain control systems were discussed in this paper. The newly proposed system introduced in this paper provides an easier way for the handicapped or ALS patients to move by a brain controlled wheelchair BCW using the technology of brain computer interface (BCI) based mobile application. Also it provides lower cost than the other systems with efficiency reached $85 \%$ of success.

\section{REFERENCES}

[1] Nikhil R Folane, Laxmikant K Shevada, Abhijeet A Chavan, Kiran R Urgunde, "Brain Computer Interface based Wheelchair: A Robotic Architecture ", International Journal of Engineering Sciences and Research Technology, IJESRT, March, 2017.

[2] Tom Carlson, Robert Leeb, Ricardo Chavarriaga and Jose del R. Millan, "The birth of the braincontrolled wheelchair", International Conference on Intelligent Robots and Systems, October 2012.

[3] Tom Carlson, Member IEEE, and Jose del R. Mill "Brain-Controlled Wheelchairs: A Robotic Architecture ",IEEE Robotics and Automation Magazine. 20(1): 65 - 73, March 2013

[4] Mrs. V. Jeyaramya A. AslineCeles, TamilNadu , TamilNadu, A. Durga Devi "Mind -Controlled Wheel Chair using an EEG Probes using Microcontroller", International Science Press,IJCTA, 9(39), 2016, pp. 19-28.

[5] YumlembamRahul , Assam DonBosco, " A Review on EEG Control Smart WheelChairA ", International Journal of Advanced Research in Computer Science, IJARCS , Volume 8, No. 9, November-December 2017.

[6] Bo Ning, Ming-jie Li , Tong Liu , Hui-min Shen, Liang Hu , Xin Fu "Human Brain Control of Electric Wheelchair with Eye-Blink Electrooculogram Signal" , International Conference on Intelligent Robotics and Applications, ICIRA 2012.

[7] K.Tanaka,K.Matsunaga,andH.Wang,"Electroencephalogram-based control of an electric wheelchair",IEEE Trans. Robotics, vol. 21, no. 4, pp. 762-766, Aug. 2005.

[8] Tsui, C. S., Jia, P., Gan, J. Q., Hu, H., \& Yuan, K.,"EMG-based hands-free wheelchair control with EOG attention shift detection", IEEE International Conference on Robotics and Biomimetic (ROBIO). doi:10.1109/robio.2007.4522346.

[9] Rebsamen, B., Burdet, E., Guan, C., Zhang, H., Teo, C. L., Zeng, Q.,...Laugier, C. (n.d.). “A BrainControlled Wheelchair Based on P300 and Path Guidance", The First IEEE/RAS-EMBS International Conference on Biomedical Robotics and Bio mechatronics, 2006. BioRob 2006. doi:10.1109/biorob.2006.1639239

[10] Carlson T, Millán JDR.” Brain-controlled wheelchairs: a robotic architecture”, IEEE Robot AutomMag. 2013;20(1):65-73.

[11] Ron-Angevin, R., Velasco-Álvarez, F., Fernández-Rodríguez, Á, Díaz-Estrella, A., Blanca-Mena, M. J., \& Vizcaíno-Martín, F. J. (2017) "Brain-Computer Interface application: auditory serial interface to control a twoclass motor-imagery-based wheelchair "Journal of NeuroEngineering and Rehabilitation, 1-17. doi:DOI 10.1186/s12984-017-0261-y. 
International Journal of Artificial Intelligence \& Applications (IJAIA) Vol.10, No.5, September 2019

[12] Shinde, N., \& George, K. (n.d.).," Brain-Controlled Driving Aid for Electric Wheelchairs”, IEEE. doi:978-1-5090-3087-3/16/\$31.00.

[13] Motor Brush Replacment - A Step by Step How To. (2011, March 23). Retrieved Nov. \&dec., 2017, from https://experimentalev.wordpress.com/2011/03/22/motor-brush-replacment-how-to/.

\section{AUTHOR}

Sherif Kamel Hussein Hassan Ratib: Graduated from the faculty of engineering in 1989 Communications and Electronics Department,Helwan University. He received his Diploma,MSc,and Doctorate in Computer Science-2007, Major Information Technology and Networking. He has been working in many private and governmental universities inside and outside Egypt for almost 15 years. He shared in the development of many industrial courses. His research interest is GSM Based Control and Macro mobility based Mobile IP. He is an Associate Professor and

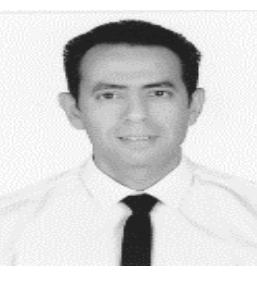
Faculty Member at Communications and Computer Engineering department in October University for Modern Sciences and Arts - Egypt. Now he is working as head of Computer Science department in Arab East Colleges for Postgraduate Studies in Riyadh- KSA. 